\title{
A NEW GAUGE MECHANISM FOR MASSIVE GAUGE BOSONS
}

\author{
DAO VONG DUC \\ Institute of Physics, VAST
}

\begin{abstract}
We propose a new mechanism for creating the mass of gauge bosons independently of Higgs mechanism. The main idea is to introduce some scalar function of space-time coordinates in the gauge transformation with some appropriate constraints. The model also allows the possibility for the gauge coupling to be changed in space-time.
\end{abstract}

\section{MOTIVATION}

The mass problem of gauge bosons has played a crucial role for the understanding of the interaction mechanism in Elementary Particle Physics. So far the Higgs mechanism[1, $2]$ has been the only one available for the gauge bosons to acquire mass. In this work we consider an alternative gauge mechanism which could also do the job. The main idea is to introduce a gauge invariance scheme involving a gauge coupling parameter which is a function of space-time coordinates.

The proposed model allows the possibility for the running physical constants to be changed in space-time and the gauge bosons to acquire masses independently of Higgs mechanism. In this context the model could be referred to as generalized (or deformed) gauge invariance.

\section{DEFORMED GAUGE INVARIANCE}

Consider first the case of $U(1)$ gauge. Let $\varphi(x)$ be some field having charge $e$ and obeying the transformation law

$$
\varphi(x) \longrightarrow \varphi^{\prime}(x)=e^{-i e \omega(x)} \varphi(x)
$$

under gauge transformation with the parameter $\omega(x)$. The covariant derivative is introduced by the formula:

$$
D_{\mu} \varphi(x)=\partial_{\mu} \varphi(x)-\operatorname{ief}(x) A_{\mu}(x) \varphi(x)
$$

where $A_{\mu}(x)$ is the gauge field with the transformation law:

$$
A_{\mu}(x) \longrightarrow A_{\mu}^{\prime}(x)=A_{\mu}(x)-\frac{1}{f(x)} \partial_{\mu} \omega(x)
$$

$f(x)$ being some scalar function parameter with some constraints to be specified later.

The equations (1) - (3) lead to the following electromagnetic interaction Lagrangians:

$$
\mathcal{L}_{\text {int }}(\psi, A)=\operatorname{ef}(x) \bar{\psi}(x) \gamma_{\mu} \psi(x) \cdot A^{\mu}(x)
$$


for spinor field $\psi$,

$$
\mathcal{L}_{i n t}(\phi, A)=e f(x) \cdot\left(\phi^{+} \partial_{\mu} \phi-\partial_{\mu} \phi^{+} \cdot \phi\right)+e^{2} f^{2}(x) \phi^{+} \phi \cdot A_{\mu} A^{\mu}
$$

for scalar field $\phi$, etc...

The conventional field strength defined by

$$
F_{\mu \nu}^{(0)} \equiv \partial_{\mu} A_{\nu}-\partial_{\nu} A_{\mu}
$$

is not invariant under the transformation (3), but its deformed expression defined by

$$
F_{\mu \nu}^{(f)} \equiv F_{\mu \nu}^{(0)}+l_{\mu} A_{\nu}-l_{\nu} A_{\mu}
$$

is invariant, and the corresponding invariant free Lagrangian for gauge field,

$$
\mathcal{L}_{0}^{(f)}(A)=-\frac{1}{4} F_{\mu \nu}^{(f)} F^{(f) \mu \nu}=-\frac{1}{4} F_{\mu \nu}^{(0)} F^{(0) \mu \nu}-\frac{1}{2}(l \cdot l)(A \cdot A)+\frac{1}{2}(l \cdot A)^{2}-F_{\mu \nu}^{(0)} l^{\mu} A^{\nu}
$$

should be used. Here we denote

$$
l_{\mu} \equiv \partial_{\mu}(\ln f)=\frac{\partial_{\mu} f}{f}, \quad(A \cdot B) \equiv A^{\mu} B_{\mu} .
$$

The Euler-Lagrange equation applied to the Lagrangian (8) gives:

$$
\left\{\square+\partial_{\nu} l^{\nu}-(l \cdot l)\right\} A_{\mu}=\left(\partial_{\mu}+l_{\mu}\right)\left(\partial^{\nu} A_{\nu}-(l \cdot A)\right)+2 \partial_{\mu} \partial_{\nu}(\ln f) \cdot A^{\nu} .
$$

Now we put the following constraint on gauge field:

$$
\partial^{\mu} A_{\mu}(x)=(l \cdot A)
$$

which could be referred to as deformed Lorentz gauge condition, and require the function parameter to be such that

$$
\partial_{\mu} \partial_{\nu}(\ln f)=\gamma \cdot \eta_{\mu \nu}, \quad \gamma=\frac{1}{4} \square(\ln f) .
$$

With the conditions (10) and (11) inserted the equation (9) becomes:

$$
\left(\square+m_{A}^{2}\right) A_{\mu}=0
$$

with the mass $m_{A}$ defined by:

$$
m_{A}^{2}=\frac{1}{2} \square(\ln f)-\frac{1}{f^{2}} \partial_{\mu} f \cdot \partial^{\mu} f .
$$

For illustration let us take an example:

$$
f(x)=e^{(p \cdot x)+\frac{r x^{2}}{2}}
$$

with $r$ being some constant, $p_{\mu}$-some real 4 -vector.

Equation (13) then gives:

$$
m_{A}^{2}=2 r-\left[p^{2}+2 r(x \cdot p)+r^{2} x^{2}\right]
$$

In particular, with $r=0$ we have:

$$
m_{A}^{2}=-p^{2}= \begin{cases}0 & \text { when } p_{0}=|\vec{p}| \\ >0 & \text { when } p_{0}<|\vec{p}|\end{cases}
$$


The results obtained above can be straightly generalized for the case of non-abelian gauge $[3,4]$.

Let $\varphi_{i}(x)$ be some field multiplet with the transformation law:

$$
\begin{aligned}
\varphi_{i}^{\prime}(x) & =(S(x))_{i}^{j} \varphi_{j}(x) \\
S(x) & =e^{-i g \sum_{a} \omega_{a}(x) M_{a}} .
\end{aligned}
$$

$M_{a}$ being representation matrices.

The covariant derivative is introduced by the formula:

$$
\begin{aligned}
D_{\mu} \varphi_{i}(x) & =\partial_{\mu} \varphi_{i}-i g f(x)\left(A_{\mu}\right)_{i}{ }^{j} \varphi_{j}, \\
A_{\mu} & =\sum_{a} A_{\mu a(x)} M_{a}
\end{aligned}
$$

with the transformation law for the gauge fields:

$$
A_{\mu}^{\prime}(x)=S A_{\mu} S^{-1}+\frac{i}{g f(x)} S \partial_{\mu} S^{-1} .
$$

The deformed expression of field strength is defined in a similar way as (7):

$$
F_{\mu \nu a}^{(f)}=\partial_{\mu} A_{\nu a}-\partial_{\nu} A_{\mu a}+g f(x) \sum_{b, c} f_{a b c} A_{\mu b} A_{\nu c}+l_{\mu} A_{\nu a}-l_{\nu} A_{\mu a} .
$$

$f_{a b c}$ being structure constants of the gauge group.

It transforms according to the formula:

$$
F_{\mu \nu a}^{(f) \prime}=S F_{\mu \nu a}^{(f)} S^{-1}, \quad F_{\mu \nu}^{(f)}=\sum_{a} F_{\mu \nu a}^{(f)} M_{a} .
$$

Hence, the invariant Lagrangian for the gauge fields is:

$$
\mathcal{L}^{(f)}(A)=-\frac{1}{2} \operatorname{Tr} F_{\mu \nu}^{(f)} F^{(f) \mu \nu}=\mathcal{L}_{0}^{(f)}(A)+\mathcal{L}_{\text {int }}^{(f)}(A),
$$

where

$$
\mathcal{L}_{0}^{(f)}(A) \equiv-\frac{1}{4} F_{\mu \nu a}^{(0)} F_{a}^{(0) \mu \nu}-\frac{1}{2}(l \cdot l)\left(A_{a} \cdot A_{a}\right)+\frac{1}{2}\left(l \cdot A_{a}\right)^{2}-F_{\mu \nu}^{(0)} \cdot l^{\mu} A_{a}^{\nu}
$$

is the free Lagrangian for gauge fields,

$$
F_{\mu \nu a}^{(0)} \equiv \partial_{\mu} A_{\nu a}-\partial_{\nu} A_{\mu a} .
$$

$\mathcal{L}_{\text {int }}^{(f)}(A)$ is the Lagrangian describing the interaction between gauge fields, it is proportional to $g f(x)$ and $(g f(x))^{2}$.

The Euler-Lagrange equation applied to the Lagrangian (21) gives:

$$
\left\{\square+\partial_{\nu} l^{\nu}-(l \cdot l)\right\} A_{\mu a}=\left(\partial_{\mu}+l_{\mu}\right)\left(\partial^{\nu} A_{\nu a}-\left(l \cdot A_{a}\right)\right)+2 \partial_{\mu} \partial_{\nu}(\ln f) \cdot A_{a}^{\nu}+g f(x)\{\cdots\} .
$$

With the same restriction (11) on the function parameter $f(x)$ and the deformed Lorentz gauge condition

$$
\partial^{\mu} A_{\mu a}=\left(l \cdot A_{a}\right) .
$$

The equation (23) becomes:

$$
\left(\square+m_{A}^{2}\right) A_{\mu a}=g f(x)\{\cdots\}
$$


with the mass $m_{A}$ defined by the same formula (13).

\section{HIGGS MECHANISM WITH DEFORMED GAUGE INVARIANCE}

In section II we have considerd a new mechanism for creating mass of gauge bosons. It is quite independent of the traditional Higgs mechanism. In this section the Higgs mechanism is treated in the framework of deformed gauge invariance described in the previous section.

Consider first the case of $U(1)$ gauge. Starting from the gauge-invariance Lagrangian

$$
\mathcal{L}=D_{\mu} \varphi^{+} \cdot D^{\mu} \phi-\alpha \phi^{+} \phi-\beta\left(\phi^{+} \phi\right)^{2}-\frac{1}{4} F_{\mu \nu}^{(f)} F^{(f) \mu \nu}, \quad \alpha<0, \beta>0,
$$

where $\phi$ is charged scalar field with non-vanishing expectation value

$$
\langle 0|\phi(x)| 0\rangle=v \equiv \sqrt{-\frac{\alpha}{2 \beta}}
$$

and performing the ordinary procedure for Higgs mechanism, we will obtain the following result for mass of the gauge boson:

$$
m_{A}^{2}=m_{A}^{(H)^{2}}+m_{A}^{(f)^{2}}
$$

where $m_{A}^{(H)}$ is the mass acquired from Higgs mechanism,

$$
m_{A}^{(H)}=-\frac{\alpha}{\beta} e^{2} f^{2}(x) .
$$

$e$ being charge of the scalar field $\varphi$, and $m_{A}^{(f)}$ is the mass acquired from the mechanism described in section II and defined by the formula (13). It is worth noting that the mass of gauge bosons could be changed in space-time.

In the case of non-abelian gauge the result is quite similar. For definiteness let us consider the case of $S U(2)$ gauge.

We start from the gauge-invariant Lagrangian

$$
\begin{aligned}
& \mathcal{L}=D_{\mu} \phi^{+} \cdot D^{\mu} \phi-\alpha \phi^{+} \phi-\beta\left(\phi^{+} \phi\right)^{2}-\frac{1}{4} \sum_{a=1} F_{\mu \nu a}^{(f)} F_{a}^{(f) \mu \nu}, \\
& D_{\mu} \phi=\partial_{\mu} \phi-i g f(x) A_{\mu a} \frac{\tau_{a}}{2} \phi, \\
& F_{\mu \nu a}^{(f)}=\partial_{\mu} A_{\nu a}-\partial_{\nu} A_{\mu a}+g f(x) \sum \varepsilon_{a b c} A_{\mu b} A_{\nu c}+l_{\mu} A_{\nu a}-l_{\nu} A_{\mu a},
\end{aligned}
$$

where $\phi$ is $S U(2)$ - doublet of scalar fields with non-vanishing expectation value

$$
\langle 0|\phi| 0\rangle=\left(\begin{array}{l}
v_{1} \\
v_{2}
\end{array}\right), \quad\left|v_{1}\right|^{2}+\left|v_{2}\right|^{2}=-\frac{\alpha}{2 \beta}
$$

and obtain:

$$
m_{A}^{2}=-\frac{\alpha}{4 \beta} g^{2} f^{2}(x)+m_{A}^{(f)^{2}}
$$




\section{CONCLUSION}

In this work we have considerd a version of gauge invariance including some spacetime function parameter with appropriate constraints, which might be called generalized (or deformed) gauge invariance. The model also allows the possibility for the gauge coupling to be changed in space-time. The results obtained might be of use for the study of the renormalization problem in Quantum Field Theory. This will be the subject of our further consideration.

\section{ACKNOWLEDGMENTS}

I would like to express my sincere thanks to the colleagues at Hanoi Institute of Physics and Hanoi National University of Education for valuable discussion and their interest.

\section{REFERENCES}

[1] P. W. Higgs, Phys. Lett. 12 (1964) 132.

[2] P. W. Higgs, Phys. Rev. 145 (1966) 1156.

[3] A. Salam, Gauge Unification of Fundamental Forces, Nobel Lecture 1979.

[4] S. L. Glashow and M. Gell - Mann, Gauge Theory of Weak and Electromagnetic Interactions, edited by C. H. Lai, World Scientific, 1981.

Received 15 July 2011. 